J. Math. Kyoto Univ.

3-1 (1963) 37-58.

\title{
The $(n+20)$-th homotopy groups of $\boldsymbol{n}$-spheres
}

Dedicated to Professor Y. Akizuki for his 60-th birthday

By

Mamoru Mimura and Hirosi Toda

(Received July 10, 1963)

This paper is a continuation of the calculation of the homotopy groups of spheres in [3]. Denote by $S^{n}$ the unit $n$-sphere in euclidean $(n+1)$-space and by $\pi_{r}\left(S^{n}\right)$ the $r$-th homotopy groups of $S^{n}$, then $\pi_{n+20}\left(S^{n}\right)$ can be calculated and our results are stated as follows.

\section{Theorem.}

$$
\begin{aligned}
& \pi_{22}\left(S^{2}\right) \cong Z_{132} \oplus Z_{2}, \\
& \pi_{23}\left(S^{3}\right) \cong Z_{2} \oplus Z_{2} \\
& \pi_{24}\left(S^{4}\right) \cong Z_{2} \oplus Z_{2} \oplus Z_{2} \oplus Z_{2} \oplus Z_{2} \oplus Z_{2}, \\
& \pi_{25}\left(S^{5}\right) \cong Z_{6} \oplus Z_{2} \oplus Z_{2}, \\
& \pi_{26}\left(S^{6}\right) \cong Z_{480} \oplus Z_{12}, \\
& \pi_{27}\left(S^{7}\right) \cong Z_{24} \\
& \pi_{28}\left(S^{8}\right) \cong Z_{24} \oplus Z_{3}, \\
& \pi_{29}\left(S^{9}\right) \cong Z_{24}, \\
& \pi_{30}\left(S^{10}\right) \cong Z_{504} \oplus Z_{24}, \\
& \pi_{31}\left(S^{11}\right) \cong Z_{24} \oplus Z_{2} \oplus Z_{2}, \\
& \pi_{32}\left(S^{12}\right) \cong Z_{24} \oplus Z_{2} \oplus Z_{2} \oplus Z_{2} \oplus Z_{2} \oplus Z_{2}, \\
& \pi_{33}\left(S^{13}\right) \cong Z_{24} \oplus Z_{2} \oplus Z_{2} \oplus Z_{2}, \\
& \pi_{34}\left(S^{14}\right) \cong Z_{240} \oplus Z_{24}, \\
& \pi_{35}\left(S^{15}\right) \cong Z_{24}, \\
& \pi_{36}\left(S^{16}\right) \cong Z_{24}, \\
& \pi_{37}\left(S^{17}\right) \cong Z_{24}, \\
& \pi_{38}\left(S^{18}\right) \cong Z_{24} \oplus Z_{12}, \\
& \pi_{39}\left(S^{19}\right) \cong Z_{24} \oplus Z_{2},
\end{aligned}
$$




$$
\begin{aligned}
& \pi_{40}\left(S^{20}\right) \cong Z_{24} \oplus Z_{2} \oplus Z_{2}, \\
& \pi_{41}\left(S^{21}\right) \cong Z_{24} \oplus Z_{2}, \\
& \pi_{42}\left(S^{22}\right) \cong Z_{24}, \\
& G_{20} \cong Z_{24} .
\end{aligned}
$$

More explicit results, for example, generators of 2-primary components are stated in (i) of $\S$ XVI. The definitions and the notations of [3] are carried over to the present work and we quote from [3] without any reference. As the continuation of [3], we give the number $\mathrm{XV}$ for the first paragraph of this paper which follows from the last chapter XIV of [3]. We shall use the notation $\pi(X, Y)$ in place of the notation $\pi(X \rightarrow Y)$ in [3].

In $\S X V$ we examine a generator $\kappa_{n}$ of $\pi_{n+14}\left(S^{n}\right)$ and calculate some secondary compositions. We show the existence of essential elements $\bar{\kappa}^{\prime}$ in $\pi_{26}\left(S^{6}\right)$ and $\bar{\kappa}_{n}$ in $\pi_{n+20}\left(S^{n}\right)$ for $n \geq 7$ in the same section.

The 2-primary components of $\pi_{n+20}\left(S^{n}\right)$, which we denote by $\pi_{n+20}^{n}$ according to [3], are calculated in (i) of XVI mainly by means of the following exact sequence :

$$
\cdots \longrightarrow \pi_{i}^{n} \stackrel{E}{\longrightarrow} \pi_{i+1}^{n+1} \stackrel{H}{\longrightarrow} \pi_{i+1}^{2 n+1} \stackrel{\Delta}{\longrightarrow} \pi_{i-1}^{n} \stackrel{E}{\longrightarrow} \pi_{i}^{n+1} \longrightarrow \cdots,
$$

where $E$ is a suspension homomorphism and. $H$ is a generalized Hopf homomorphism. To complete the calculation of $\pi_{n+20}\left(S^{n}\right)$ we compute the odd primary components of $\pi_{n+20}\left(S^{n}\right)$ in (ii) of $\S \mathrm{XVI}$.

\section{$\S \mathrm{XV}$. Some elements of $\pi_{n+20}\left(S^{n}\right)$.}

In chapter $X$ of [3], we have given elements

$$
\kappa_{n}=E^{n-7} \kappa_{7} \quad(n \geq 7), \quad \bar{\varepsilon}_{n}=E^{n-3} \bar{\varepsilon}_{3} \quad(n \geq 3),
$$

which have the following properties:

$$
2 \kappa_{7} \equiv \bar{\nu}_{7} \circ \nu_{15}^{2} \quad \bmod 4\left(\sigma^{\prime} \circ \sigma_{14}\right) .
$$

$$
\left\{\varepsilon_{3}, 2 \iota_{11}, \nu_{11}^{2}\right\}_{6} \quad \text { consists of the single element } \bar{\varepsilon}_{3} \text {. }
$$

$$
\begin{array}{ll}
\eta_{n} \circ \kappa_{n+1}=\bar{\varepsilon}_{n} & \text { for } n \geq 6 \text { and } \\
\kappa_{n} \circ \eta_{n+14}=\bar{\varepsilon}_{n} & \text { for } n \geq 9 .
\end{array}
$$

$$
\eta_{n}^{2} \circ \kappa_{n+2}=\kappa_{n} \circ \eta_{n+14}^{2}=0 \quad \text { for } n \geq 9 \text {. }
$$


The relation (15.1) follows from Lemma 10.1, (10.7) and Lemma 5.14. By (4.7) and Theorem 10.5, $\left\{\varepsilon_{3}, 2 \iota_{11}, \nu_{11}^{2}\right\}_{6} \subset \pi_{18}^{3}=$ $\left\{\bar{\varepsilon}_{3}\right\} \cong Z_{2}$. But the proof of Lemma 10.2 shows that $H H\left\{\varepsilon_{3}, 2 \iota_{11}, \nu_{11}^{2}\right\}_{6}$ $=\nu_{9}^{3} \neq 0$. Thus $\left\{\varepsilon_{3}, 2 \iota_{11}, \nu_{11}^{2}\right\}_{6} \neq 0$ and (15.2) follows. (15.3) is (10.23). (15.4) follows from Lemma 12.10, the relation $\Delta\left(\nu_{17}^{3}\right)=\nu_{8} \circ \sigma_{11} \circ \nu_{18}^{3}$ of page 142 and from the exactness of $\pi_{26}^{17} \stackrel{\Delta}{\longrightarrow} \pi_{24}^{8} \stackrel{E}{\longrightarrow} \pi_{25}^{9} \longrightarrow 0$. It follows from (15.1) that

$$
\begin{aligned}
& 2 \kappa_{7}=\bar{\nu}_{7} \circ \nu_{15}^{2} \quad \text { or } \\
& 2\left(\kappa_{7}+2 \sigma^{\prime} \circ \sigma_{14}\right)=\bar{\nu}_{7} \circ \nu_{15}^{2} .
\end{aligned}
$$

Remark that the next relation holds :

$$
\kappa_{7} \circ \eta_{21}=\sigma^{\prime} \circ \bar{\nu}_{14}+\bar{\varepsilon}_{7} .
$$

Now we change the definition of $\kappa_{7}$. We replace $\kappa_{7}$ by $\kappa_{7}+2 \sigma^{\prime} \circ \sigma_{14}$ in the second case of $(15.1)^{\prime}$. Then we have

$$
\begin{array}{ll}
2 \kappa_{7}=\bar{\nu}_{7} \circ \nu_{15}^{2} & \text { and } \\
2 \kappa_{n}=2 \iota_{n} \circ \kappa_{n}=0 & \text { for } n \geq 10 .
\end{array}
$$

The second part of the above relation follows from (15.1)' and that $\bar{\nu}_{n} \circ \nu_{n+8}=0$ for $n \geq 10$ (cf. (7.22)). It is easily checked that the relations (15.3) and (15.4) still hold for new $\kappa_{7}$ and $\kappa_{n}=E^{n_{-7}} \kappa_{7}, n \geq 7$.

We shall prove the following lemmas.

Lemma 15. 1. For $n \geq 9$, we have

$$
\left\{\eta_{n}, \eta_{n+1} \circ \kappa_{n+2}, 2 \iota_{n+16}\right\}_{n-5} \equiv \nu_{n} \circ \kappa_{n+3}
$$

$\bmod 2 \pi_{n+17}\left(S^{n}\right)+\eta_{n} \circ E^{n-5} \pi_{22}^{6}+\left\{\eta_{n} \circ \mu_{n+1} \circ \sigma_{n+10}+\sigma_{n} \circ \eta_{n+7} \circ \mu_{n+8}\right\}$,

where $\quad 2 \pi_{n+17}\left(S^{n}\right)=0$ if $n \neq 14(n \geq 9)$,

$$
\begin{aligned}
& \eta_{n} \circ E^{n-5} \pi_{22}^{6}+\left\{\eta_{n} \circ \mu_{n+1} \circ \sigma_{n+10}+\sigma_{n} \circ \eta_{n+7} \circ \mu_{n+8}\right\} \\
& =\left\{\eta_{n} \circ \mu_{n+1} \circ \sigma_{n+10}\right\} \\
& \text { if } n \geq 10 .
\end{aligned}
$$

Lemma 15. 2. For $n \geq 12$, we have

$$
\left\{\eta_{n}, 2 \iota_{n+1}, \kappa_{n+1}\right\}_{n-9} \equiv 0 \quad \bmod \eta_{n} \circ \rho_{n+1} .
$$

Proof of Lemma 15.1. Let $n$ be sufficiently large such that 
the homotopy groups in the following discussions are stable. (e.g., $n \geq 19$.) The secondary composition $\left\{\nu_{n}^{2}, 2 \iota_{n+6}, \nu_{n+6}^{2}\right\}$ is defined and it is a subset of $\pi_{n+13}^{n}$. Since $\pi_{n+13}^{n}=0$ for $n \geq 15$ (Theorem 7.7), $\left\{\nu_{n}^{2}, 2 \iota_{n+6}, \nu_{n+6}^{2}\right\}=0$. We have also $\left\{2 \iota_{n}, \nu_{n}^{2}, 2 \iota_{n+6}\right\} \equiv \nu_{n 0}^{2} \circ \eta_{n+6}=0$ by Corollary 3.7 and (5.9).

Let a cell complex $K=S^{n} \cup e^{n_{+}}$has the characteristic class $\nu_{n}^{2}$ of $e^{n_{+}}$. By Proposition 1.7, there exists an extension $\operatorname{Ext}\left(2 \iota_{n}\right) \epsilon$ $\pi\left(K, S^{n}\right)$ of $2 \iota_{n}$ and a coextension Coext $\left(2 \iota_{n+6}\right) \in \pi_{n+7}(K)$ of $2 \iota_{n+6}$, such that $\operatorname{Ext}\left(2 \iota_{n}\right) \circ \operatorname{Coext}\left(2 \iota_{n+6}\right)=0$. By Proposition 1. 8, Coext $\left(2 \iota_{n+6}\right) \circ \nu_{n+7}^{2}$ $\in i^{*}\left\{\nu_{n}^{2}, 2 \iota_{n+6}, \nu_{n+6}^{2}\right\}=0$ where $i$ is the injection of $S^{n}$ into $K$. Thus the following secondary composition is defined.

$$
\left\{\operatorname{Ext}\left(2 \iota_{n}\right), \operatorname{Coext}\left(2 \iota_{n+6}\right), \nu_{n+7}^{2}\right\}<\pi_{n+14}\left(S^{n}\right)
$$

Consider the composition with $\eta_{n-1}$, then

$$
\begin{aligned}
& \eta_{n-1} \circ\left\{\operatorname{Ext}\left(2 \iota_{n}\right), \operatorname{Coext}\left(2 \iota_{n+6}\right), \nu_{n+7}^{2}\right\} \\
& \subset\left\{\eta_{n-1} \circ \operatorname{Ext}\left(2 \iota_{n}\right), \text { Coext }\left(2 \iota_{n+6}\right), \nu_{n+7}^{2}\right\} \quad \text { by Proposition } 1.2 \\
& \subset\left\{p^{*}\left\{\eta_{n-1}, 2 \iota_{n}, \nu_{n}^{2}\right\}, \text { Coext }\left(2 \iota_{n+6}\right), \nu_{n+7}^{2}\right\} \quad \text { by Proposition } 1.9 \\
& =\left\{p^{*}\left(\varepsilon_{n-1}+\left\{\eta_{n-1} \circ \sigma_{n}\right\}\right), \text { Coext }\left(2 \iota_{n+6}\right), \nu_{n+7}^{2}\right\} \quad \text { by (6.1) } \\
& C\left\{\varepsilon_{n-1}+\left\{\eta_{n-1} \circ \sigma_{n}\right\}, p_{*} \operatorname{Coext}\left(2 \iota_{n+6}\right), \nu_{n+7}^{2}\right\} \quad \text { by Proposition } 1.2 \\
& C\left\{\varepsilon_{n-1}, 2 \iota_{n+7}, \nu_{n+7}^{2}\right\}+\left\{\eta_{n-1} \circ \sigma_{n}, 2 \iota_{n+7}, \nu_{n+7}^{2}\right\} \quad \text { by (1. 18), }
\end{aligned}
$$

where $p: K \rightarrow S^{n ! 7}$ is a mapping which shrinks $S^{n}$ to a point. Here we have

$$
\begin{aligned}
\left\{\varepsilon_{n-1}, 2 \iota_{n+7}, \nu_{n+7}^{2}\right\} & \supset E^{n-4}(-1)^{n}\left\{\varepsilon_{3}, 2 \iota_{11}, \nu_{11}^{2}\right\}_{6} & & \text { by Proposition } 1.3 \\
\ni & E^{n-4}\left((-1)^{n} \bar{\varepsilon}_{3}\right)=\bar{\varepsilon}_{n-1} & & \text { by definitions, } \\
\left\{\eta_{n-1} \circ \sigma_{n}, 2 \iota_{n+7}, \nu_{n+7}^{2}\right\} & =\left\{\sigma_{n-1} \circ \eta_{n+6}, 2 \iota_{n+7}, \nu_{n+7}^{2}\right\} & & \\
& \supset \sigma_{n-1} \circ\left\{\eta_{n+6}, 2 \iota_{n+7}, \nu_{n+7}^{2}\right\} & & \text { by Proposition } 1.2 \\
& \ni \sigma_{n-1} \circ \varepsilon_{n+6} & & \text { by }(7.1) \\
& =0 & & \text { by Lemma } 10.7 .
\end{aligned}
$$

These secondary compositions are cosets of sums of $\eta_{n-1} \circ \sigma_{n} \circ \pi_{n+14}^{n+7}=$ $\eta_{n-1} \circ \sigma_{n} \circ\left\{\sigma_{n+7}\right\}, \quad \varepsilon_{n-1} \circ \pi_{n+14}^{n+7}=\varepsilon_{n-1} \circ\left\{\sigma_{n+7}\right\} \quad$ and $\pi_{n+8}^{n-1} \circ \nu_{n+8}^{2}=\left\{\nu_{n-1}^{3}, \mu_{n-1}\right.$, $\left.\eta_{n-1} \circ \varepsilon_{n}\right\} \circ \nu_{n+8}^{2}$, which are zeros by the relations (14.1):

$$
\eta_{n-1} \circ \sigma_{n}^{2}=0, \quad \varepsilon_{n-1} \circ \sigma_{n+7}=0, \quad \nu_{n-1}^{3} \circ \nu_{n+8}^{2}=0, \quad \mu_{n-1} \circ \nu_{n+8}^{2}=0
$$

and $\eta_{n-1} \circ \varepsilon_{n} \circ \nu_{n+8}^{2}=0$. 
Therefore we obtain

$$
\eta_{n-1} \circ\left\{\operatorname{Ext}\left(2 \iota_{n}\right), \operatorname{Coext}\left(2 \iota_{n+6}\right), \nu_{n+7}^{2}\right\}=\bar{\varepsilon}_{n-1}=\eta_{n-1} \circ \kappa_{n} .
$$

As the kernel of $\left(\eta_{n-1}\right)_{*}: \pi_{n+14}\left(S^{n}\right) \rightarrow \pi_{n+14}\left(S^{n-1}\right)$ is generated by $\sigma_{n}^{2}$, so $\kappa_{n}$ or $\kappa_{n}+\sigma_{n}^{2}$ belongs to $\left\{\operatorname{Ext}\left(2 \iota_{n}\right)\right.$, Coext $\left.\left(2 \iota_{n+6}\right), \nu_{n+7}^{2}\right\}$. Since $\sigma_{n}^{2} \circ \nu_{n+14}=0$ by $(7.20)$, we have

$$
\begin{aligned}
\kappa_{n} \circ \nu_{n+14} & \in\left\{\operatorname{Ext}\left(2 \iota_{n}\right), \operatorname{Coext}\left(2 \iota_{n+6}\right), \nu_{n+7}^{2}\right\} \circ \nu_{n+14} & & \\
& <\left\{\operatorname{Ext}\left(2 \iota_{n}\right), \operatorname{Coext}\left(2 \iota_{n+6}\right), \nu_{n+7}^{3}\right\} & & \text { by Proposition 1.2 } \\
& =\left\{\operatorname{Ext}\left(2 \iota_{n}\right), \operatorname{Coext}\left(2 \iota_{n+6}\right), \bar{\nu}_{n+7} \circ \eta_{n+15}\right\} & & \text { by Lemma 6. } \\
& \subset\left\{\operatorname{Ext}\left(2 \iota_{n}\right), \operatorname{Coext}\left(2 \iota_{n+6}\right) \circ \bar{\nu}_{n+7}, \eta_{n+15}\right\} & & \text { by Proposition 1.2 } \\
& =\left\{\operatorname{Ext}\left(2 \iota_{n}\right),-\operatorname{Coext}\left(2 \iota_{n+6}\right) \circ \bar{\nu}_{n+7}, \eta_{n+15}\right\} . & &
\end{aligned}
$$

Here - Coext $\left(2 \iota_{n+6}\right) \circ \bar{\nu}_{n+7}=i^{*}\left\{\nu_{n}^{2}, 2 \iota_{n+6}, \bar{\nu}_{n+6}\right\} \quad$ by Proposition 1.8

$$
\begin{aligned}
& =i_{*} \bar{\varepsilon}_{n} \quad c f \text {. page } 111 \\
& =i_{*}\left(\eta_{n} \circ \kappa_{n+1}\right) \quad \text { by }(15.3) \text {. }
\end{aligned}
$$

Therefore

$$
\begin{aligned}
\kappa_{n} \circ \nu_{n+14} & \in\left\{\operatorname{Ext}\left(2 \iota_{n}\right), i_{*}\left(\eta_{n} \circ \kappa_{n+1}\right), \eta_{n+15}\right\} \\
& \supset\left\{i * \operatorname{Ext}\left(2 \iota_{n}\right), \eta_{n} \circ \kappa_{n+1}, \eta_{n+15}\right\} \\
& =\left\{2 \iota_{n}, \eta_{n} \circ \kappa_{n+1}, \eta_{n+15}\right\},
\end{aligned}
$$

by Proposition 1.2

where $i_{*}$ and $i^{*}$ are induced by the injection $i$. The composition $\left\{2 \iota_{n}, \eta_{n} \circ \kappa_{n+1}, \eta_{n+15}\right\}$ is a coset of $2 \pi_{n+17}\left(S^{n}\right)+\pi_{n+16}^{n} \circ \eta_{n+16}$, where $2 \pi_{n+17}\left(S^{n}\right)=0$ by the fact that $\pi_{n+17}\left(S^{n}\right)$ does not have the odd primary components and the 2-primary component of it is $Z_{2} \oplus Z_{2} \oplus Z_{2} \oplus Z_{2}$ and $\pi_{n+16}^{n} \circ \eta_{n+16}=\left\{\eta_{n}^{*} \circ \eta_{n+16}, \eta_{n} \circ \mu_{n+1} \circ \sigma_{n+10}\right\} . \quad\left\{\operatorname{Ext}\left(2 \iota_{n}\right)\right.$, $\left.i_{*}\left(\eta_{n} \circ \kappa_{n+1}\right), \eta_{n+15}\right\}$ is a coset of $\pi_{n+16}^{n} \circ \eta_{n+16}+\operatorname{Ext}\left(2 \iota_{n}\right) \circ \pi_{n+17}(K)$. When $n$ is sufficiently large, the elements of $\pi_{n+17}(K)$ are considered as the coextensions of elements of $\pi_{n+16}\left(S^{n+6}\right)$. So $\operatorname{Ext}\left(2 \iota_{n}\right) \circ \pi_{n+17}(K)$ is the set of all $\operatorname{Ext}\left(2 \iota_{n}\right) \circ \operatorname{Coext}(\beta)$, where $\beta \in \pi_{n+16}\left(S^{n+6}\right) \cong Z_{6}$ and $3 \beta=0$ or $3 \beta=\eta_{n+6} \circ \mu_{n+7}$.

By Proposition 1.7,

$$
\begin{array}{rlrl}
\operatorname{Ext}\left(2 \iota_{n}\right) \circ \operatorname{Coext}(\beta) & =\left\{2 \iota_{n}, \nu_{n}^{2}, \beta\right\} & & \text { by Proposition } 1.7 \\
& =\left\{2 \iota_{n}, 3 \nu_{n}^{2}, \beta\right\} & \\
& >\left\{2 \iota_{n}, \nu_{n}^{2}, 3 \beta\right\} & \text { by Proposition } 1.2 \\
& =\left\{2 \iota_{n}, \nu_{n}^{2}, 0\right\} \text { or }\left\{2 \iota_{n}, \nu_{n}^{2}, \eta_{n+6} \circ \mu_{n+7}\right\}
\end{array}
$$


where $\left\{2 \iota_{n}, \nu_{n}^{2}, 0\right\}=0$ by $2 \pi_{n+17}\left(S^{n}\right)=0$ and

$$
\begin{aligned}
\left\{2 \iota_{n}, \nu_{n}^{2}, \eta_{n+6} \circ \mu_{n+7}\right\} & \supset\left\{2 \iota_{n}, \nu_{n}^{2}, \eta_{n+6}\right\} \circ \mu_{n+8} & & \\
& \ni \varepsilon_{n} \circ \mu_{n+8} & & \text { by }(6.1) \text { and (3.9) } \\
& =\left(\bar{\nu}_{n}+\sigma_{n} \circ \eta_{n+7}\right) \circ \mu_{n+8} & & \text { by Lemma } 6.4 \\
& =\sigma_{n} \circ \eta_{n+7} \circ \mu_{n+8} & & \text { by Theorem 14. 1. }
\end{aligned}
$$

Therefore

$$
\begin{aligned}
\operatorname{Ext}\left(2 \iota_{n}\right) \circ \operatorname{Coext}(\beta) & <2 \pi_{n+17}\left(S^{n}\right)+\pi_{n+7}\left(S^{n}\right) \circ \beta+\left\{\sigma_{n} \circ \eta_{n+7} \circ \mu_{n+8}\right\} \\
= & \left\{\sigma_{n} \circ \eta_{n+7} \circ \mu_{n+8}\right\} \\
& <\pi_{n+16}^{n} \circ \eta_{n+16} .
\end{aligned}
$$

Thus $\left\{\operatorname{Ext}\left(2 \iota_{n}\right), i_{*}\left(\eta_{n} \circ \kappa_{n+1}\right), \eta_{n+15}\right\}$ and $\left\{2 \iota_{n}, \eta_{n} \circ \kappa_{n+1}, \eta_{n+15}\right\}$ are the same coset of the subgroup $\pi_{n+16}^{n} \circ \eta_{n+16}$ of $\pi_{n+17}^{n}$. Whence, for a sufficiently large $n$,

$$
\begin{aligned}
\nu_{n} \circ \kappa_{n+3}=\kappa_{n} \circ \nu_{n+14} & \in\left\{2 \iota_{n}, \eta_{n} \circ \kappa_{n+1}, \eta_{n+15}\right\} \\
& =\left\{\eta_{n}, \eta_{n+1} \circ \kappa_{n+2}, 2 \iota_{n+16}\right\} \quad \text { by (3.9). }
\end{aligned}
$$

Now the lemma follows from the fact that the odd primary components of $\pi_{n+17}\left(S^{n}\right)$ are zero for $n \geq 9, n \neq 14$ and that the kernel of $E^{n-9}: \pi_{26}^{9} \rightarrow \pi_{n+17}^{n}$ is generated by $\sigma_{9} \circ \eta_{16} \circ \mu_{17}+\eta_{9} \circ \mu_{10} \circ \sigma_{19}$ (cf. Theorem 12.7, 12.17 and Ch. XIV). q.e.d.

Proof of Lemma 15.2. Consider the secondary composition $\left\{\eta_{12}, 2 \iota_{13}, \kappa_{13}\right\}_{3}<\pi_{28}^{12}$ which can be defined since the order of $\eta_{12}$ and $\kappa_{10}$ are 2. This composition is a coset of the subgroup

$$
\eta_{12} \circ E^{3} \pi_{25}^{1 n}+\pi_{14}^{12} \circ \kappa_{14}=\left\{\eta_{12} \circ E^{4} \rho^{\prime}, \eta_{12} \circ \sigma_{13} \circ \bar{\nu}_{20}, \eta_{12} \circ \bar{\varepsilon}_{13}, \eta_{12}^{2} \circ \kappa_{14}\right\},
$$

where $\quad \eta_{12} \circ E^{4} \rho^{\prime}=\eta_{12} \circ 2 \rho_{13}=2\left(\eta_{12} \circ \rho_{13}\right)=0 \quad$ by Lemma 10.9

$$
\eta_{12} \circ \sigma_{13} \circ \bar{\nu}_{20}=0 \quad \text { by }(10.18)
$$

and $\quad \eta_{12} \circ \bar{\varepsilon}_{13}=\eta_{12}^{2} \circ \kappa_{14}=0 \quad$ by (15.3) and (15.4).

So it consists of a single element. As $\pi_{28}^{12}$ is generated by $\sigma_{12} \circ \mu_{19}$, so $\begin{aligned}\left\{\eta_{12}, 2 \iota_{13}, \kappa_{13}\right\}_{3} & =a\left(\sigma_{12} \circ \mu_{19}\right) & & \text { where } a=0,1 \\ & =a\left(\eta_{12} \circ \rho_{13}\right) & & \text { by Proposition } 12.20 .\end{aligned}$

Therefore, if $n \geq 12$,

$$
\left\{\eta_{n}, 2 \iota_{n+1}, \kappa_{n+1}\right\}_{n-9}>E^{n-12}\left\{\eta_{12}, 2 \iota_{13}, \kappa_{13}\right\}_{3} \ni E^{n-12}\left(a \eta_{12} \circ \rho_{13}\right)=a\left(\eta_{n} \circ \rho_{n+1}\right) \text {. }
$$


This concludes the lemma.

q.e.d.

Lemma 15. 3. There exists an element $\bar{\kappa}^{\prime}$ of $\pi_{26}\left(S^{6}\right)$ such that

$$
2 \bar{\kappa}^{\prime}=\nu_{6}^{2} \circ \kappa_{12} \text { and } 4 \bar{\kappa}^{\prime}=0 .
$$

Proof. By the relation $\nu_{6}^{\circ} \eta_{9}=0(c f .(5.9))$ and (15.4), we can define a secondary composition $\left\{\nu_{6}, \eta_{9}, \eta_{10} \circ \kappa_{11}\right\}$, from which we choose an element $\bar{\kappa}^{\prime}$.

$$
\begin{aligned}
2 \bar{\kappa}^{\prime}=\bar{\kappa}^{\prime} \circ 2 \iota_{26} & \in\left\{\nu_{6}, \eta_{9}, \eta_{10} \circ \kappa_{11}\right\} \circ 2 \iota_{26} \\
& =-\nu_{6} \circ\left\{\eta_{9}, \eta_{10} \circ \kappa_{11}, 2 \iota_{25}\right\} \quad \text { by Proposition 1.4. }
\end{aligned}
$$

On the other hand, by Lemma 15.1 for $n=9$, we have

$$
\begin{array}{rlrl}
\nu_{6} \circ\left\{\eta_{9}, \eta_{10} \circ \kappa_{11}, 2 \iota_{25}\right\}_{4} \equiv \nu_{6}^{2} \circ \kappa_{12} & \\
\bmod \nu_{6} \circ \eta_{9} \circ E^{4} \pi_{22}^{6}+\left\{\nu_{6} \circ \eta_{9} \circ \mu_{10} \circ \sigma_{19}+\nu_{6} \circ \sigma_{9} \circ \eta_{16} \circ \mu_{17}\right\}, \\
\nu_{6} \circ \eta_{9} & =0 & & \text { by }(5.9) \text { and } \\
\nu_{6} \circ \sigma_{9} \circ \eta_{16} \circ \mu_{17} & =\nu_{6} \circ \varepsilon_{9} \circ \mu_{17} & & \text { (see page 152) } \\
& =2\left(\bar{\nu}_{6} \circ \nu_{14}\right) \circ \mu_{17} & & \text { by }(7.18) \\
& =0 . &
\end{array}
$$

where

That is,

$$
\begin{aligned}
\nu_{6}^{2} \circ \kappa_{12} & =\nu_{6} \circ\left\{\eta_{9}, \eta_{10} \circ \kappa_{11}, 2 \iota_{25}\right\}_{4} \\
& <\nu_{6} \circ\left\{\eta_{9}, \eta_{10} \circ \kappa_{11}, 2 \iota_{25}\right\}=-\left\{\nu_{6}, \eta_{9}, \eta_{10} \circ \kappa_{11}\right\} \circ 2 \iota_{26} .
\end{aligned}
$$

The last composition is a coset of

$$
\begin{gathered}
\nu_{6} \circ \pi_{26}\left(S^{9}\right) \circ 2 \iota_{26}=\nu_{6} \circ 2 \pi_{26}\left(S^{9}\right)=0, \text { since } \pi_{26}\left(S^{9}\right) \cong Z_{2} \oplus Z_{2} \oplus Z_{2} \oplus Z_{2} . \\
\begin{array}{l}
\text { Therefore } \\
2 \bar{\kappa}^{\prime}=-\nu_{6}^{2} \circ \kappa_{12}=\nu_{6}^{2} \circ \kappa_{12} .
\end{array}
\end{gathered}
$$

This implies that $4 \bar{\kappa}^{\prime}=2 \nu_{6}^{2} \circ \kappa_{12}=0$.

q.e.d.

Consider a cell-complex $L=S^{13} \cup e^{14}$ where $e^{14}$ is attached to $S^{13}$ by a mapping of degree 2. Apply Proposition 1.7 to Lemma 15.2, then we have an extension $\operatorname{Ext}\left(\eta_{12}\right) \in \pi\left(L, S^{12}\right)$ of $\eta_{12}$ and a coextension Coext $\left(\kappa_{13}\right) \in \pi_{18}(L)$ of $\kappa_{13}$, such that $\operatorname{Ext}\left(\eta_{12}\right) \circ \operatorname{Coext}\left(\kappa_{13}\right)=0$. By Proposition 1.9, Proposition 5.9 and Theorem 13.4,

$$
\begin{aligned}
\nu_{9} \circ \operatorname{Ext}\left(\eta_{12}\right) & \in\left(E^{5} p\right)^{*}\left\{\nu_{9}, \eta_{12}, 2 \iota_{13}\right\}_{9} \\
& \subset\left(E^{5} p\right)^{*} \pi_{14}\left(S^{9}\right)=0,
\end{aligned}
$$


where $p: S^{4} \cup e^{5} \rightarrow S^{5}$ is a mapping which shrinks $S^{4}$ to a point. Therefore we can define a secondary composition $\left\{\nu_{9}, \operatorname{Ext}\left(\eta_{12}\right)\right.$, Coext $\left.\left(\kappa_{13}\right)\right\}$. We choose an element $\bar{\kappa}_{9}$ from this. Then we have

$$
\begin{aligned}
2 \bar{\kappa}_{9} & \in\left\{\nu_{9}, \operatorname{Ext}\left(\eta_{12}\right), \operatorname{Coext}\left(\kappa_{13}\right)\right\} \circ 2 \iota_{29} \\
& \subset\left\{\nu_{9}, \operatorname{Ext}\left(\eta_{12}\right), \operatorname{Coext}\left(\kappa_{13}\right) \circ 2 \iota_{28}\right\} \\
& \subset\left\{\nu_{9}, \operatorname{Ext}\left(\eta_{12}\right),-i_{*}\left\{2 \iota_{13}, \kappa_{13}, 2 \iota_{27}\right\}_{3}\right\} \quad \text { by Proposition } 1.2
\end{aligned}
$$

As $E^{n-13}: \pi_{28}^{13} \rightarrow \pi_{n+15}^{n}$ is an isomorphism onto and $\left\{2 \iota_{n}, \kappa_{n}, 2 \iota_{n+14}\right\}_{n-10}$ contains an element $\kappa_{n} \circ \eta_{n+14}=\eta_{n} \circ \kappa_{n+1}$ by Corollary 3. 7, so $\left\{2 \iota_{13}, \kappa_{13}\right.$, $\left.2 \iota_{27}\right\}_{3}$ contains the element $\kappa_{13} \circ \eta_{27}=\eta_{13} \circ \kappa_{14}$. Here, the composition $\left\{2 \iota_{13}, \kappa_{13}, 2 \iota_{27}\right\}_{3}$ is a coset of $2 \iota_{13} \circ E^{3} \pi_{15}\left(S^{10}\right)+\pi_{28}\left(S^{13}\right) \circ 2 \iota_{28}=2\left(\pi_{28}\left(S^{13}\right)\right)$. We have $2 \pi_{28}\left(S^{13}\right)=2 \iota_{13} \circ \pi_{28}\left(S^{13}\right)$, by use of the isomorphism $E: \pi_{28}\left(S^{13}\right)$ $\rightarrow \pi_{29}\left(S^{14}\right)$. As $i_{*}\left(2 \iota_{13}\right)=0, i_{*}\left\{2 \iota_{13}, \kappa_{13}, 2 \iota_{17}\right\}_{3}$ consists of the single element $i_{*}\left(\kappa_{13} \circ \eta_{27}\right)=i_{*}\left(\eta_{13} \circ \kappa_{14}\right)$. Therefore we have

$$
\begin{aligned}
2 \bar{\kappa}_{9} & \in\left\{\nu_{9}, \operatorname{Ext}\left(\eta_{12}\right),-i_{*}\left(\eta_{13} \circ \kappa_{14}\right)\right\} \\
& =\left\{\nu_{9}, \operatorname{Ext}\left(\eta_{12}\right), i_{*}\left(\eta_{13} \circ \kappa_{14}\right)\right\} \\
& \subset\left\{\nu_{9}, i * \operatorname{Ext}\left(\eta_{12}\right), \eta_{13} \circ \kappa_{14}\right\} \\
& =\left\{\nu_{9}, \eta_{12}, \eta_{13} \circ \kappa_{14}\right\}
\end{aligned}
$$$$
\supset\left\{\nu_{9}, \eta_{12}, \eta_{13} \circ \kappa_{14}\right\}_{3} \ni E^{3} \bar{\kappa}^{\prime} \quad \text { by Lemma 15.3. }
$$

Therefore $\quad 2 \bar{\kappa}_{9} \equiv E^{3} \kappa^{\prime} \quad \bmod \nu_{9} \circ \pi_{29}\left(S^{12}\right)+\pi_{14}^{9} \circ \eta_{14} \circ \kappa_{15}$.

So $\quad 4 \bar{\kappa}_{9} \equiv 2 E^{3} \bar{\kappa}^{\prime} \quad \bmod \nu_{9} \circ 2 \pi_{29}\left(S^{12}\right)+\pi_{14}^{9} \circ 2\left(\eta_{14} \circ \kappa_{15}\right)=0$

$$
=\nu_{9}^{2} \circ \kappa_{15} \quad \text { by Lemma 15. } 3 \text {. }
$$

Lemma 4.5 and the exactness of $\pi_{27}^{7} \rightarrow \pi_{28}^{8} \rightarrow \pi_{28}^{15}=0$ imply that $E: \pi_{27}^{7} \rightarrow \pi_{28}^{8}$ is an isomorphism onto. That $E: \pi_{28}^{8} \rightarrow \pi_{29}^{9}$ is an isomorphism onto follows from the exact sequence $0=\pi_{30}^{17} \stackrel{\Delta}{\longrightarrow} \pi_{28}^{8}$ $\stackrel{E}{\longrightarrow} \pi_{29}^{9} \stackrel{H}{\longrightarrow} \pi_{29}^{17}=0$.

By these facts, there exists an element $\kappa_{7} \in \pi_{27}^{7}$ such that $4 \bar{\kappa}_{7}=2 E \bar{\kappa}^{\prime}=\nu_{7}^{2} \circ \kappa_{13}$ and $8 \bar{\kappa}_{7}=0$. Thus we have obtained

Lemma 15. 4. There exists an element $\bar{\kappa}_{7}$ of $\pi_{27}^{7}$ such that

$$
4 \bar{\kappa}_{7}=2 E \bar{\kappa}^{\prime}=\nu_{7}^{2} \circ \kappa_{13} \text { and } 8 \bar{\kappa}_{7}=0 .
$$

Hereafter we denote $\bar{\kappa}_{n}=E^{n-7} \bar{\kappa}_{7}$ for $n \geq 7$ and $E^{\infty} \bar{\kappa}_{7}=\bar{\kappa}$. 
$\S$ XVI. Computation of $\boldsymbol{\pi}_{n+20}\left(\boldsymbol{S}^{n}\right)$.

(i) 2-primary components of $\pi_{n+20}\left(S^{n}\right)$.

We shall compute the 2-primary components of $\pi_{n+20}\left(S^{n}\right)$. First we have

$$
\pi_{22}^{2}=\left\{\eta_{2} \circ \bar{\mu}^{\prime}, \eta_{2} \circ \nu^{\prime} \circ \mu_{6} \circ \sigma_{15}\right\} \simeq Z_{4} \oplus Z_{2}
$$

by (5.2) and Theorem 12.9.

Consider the exact sequence

$$
\text { where } \quad \cdots \longrightarrow \pi_{24}^{5} \stackrel{\Delta}{\longrightarrow} \pi_{22}^{2} \stackrel{E}{\longrightarrow} \pi_{23}^{3} \stackrel{H}{\longrightarrow} \pi_{23}^{5} \stackrel{\Delta}{\longrightarrow} \pi_{21}^{2} \longrightarrow \cdots,
$$

We have in the page 149 of [3]

$$
\begin{aligned}
& \Delta\left(\zeta_{5} \circ \sigma_{16}\right) \equiv \pm\left(\eta_{2} \circ \mu^{\prime} \circ \sigma_{14}\right) \bmod \left\{\eta_{2} \circ \nu^{\prime} \circ \bar{\varepsilon}_{6}\right\}+\left\{\eta_{2}^{2} \circ \bar{\mu}_{4}\right\}, \\
& \Delta\left(\nu_{5} \circ \bar{\varepsilon}_{8}\right)=\eta_{2} \circ \nu^{\circ} \circ \bar{\varepsilon}_{6}, \\
& \Delta\left(\eta_{5} \circ \bar{\mu}_{6}\right)=0 .
\end{aligned}
$$

Then we have $H \pi_{23}^{3}=\operatorname{Ker} \Delta=\left\{\eta_{5} \circ \bar{\mu}_{6}, 4 \zeta_{5} \circ \sigma_{16}\right\} \cong Z_{2} \oplus Z_{2}$.

$$
\begin{aligned}
& \text { And } E\left(\eta_{2} \circ \nu^{\prime} \circ \mu_{6} \circ \sigma_{15}\right)=0 \\
& \text { by Lemma } 5.7 \\
& E^{2}\left(\eta_{2} \circ \bar{\mu}^{\prime}\right)=\eta_{4} \circ E^{2}\left(\bar{\mu}^{\prime}\right) \\
& =\eta_{4} \circ\left(2 \bar{\zeta}_{5}\right) \quad \text { by Lemma } 12.4 \\
& =\eta_{4} \circ 2 \iota_{5} \circ \bar{\zeta}_{5} \text { by use of the monomorphism } E: \pi_{24}^{5} \rightarrow \pi_{25}^{6} \\
& =0 \text {. }
\end{aligned}
$$

So, by the fact that $E: \pi_{23}^{3} \rightarrow \pi_{24}^{4}$ is a monomorphism, we have

$$
E\left(\eta_{2} \circ \overline{\mu b}^{\prime}\right)=0 \text {. }
$$

Thus

$$
H: \pi_{23}^{3} \approx H \pi_{23}^{3}=\operatorname{Ker} \Delta .
$$

Since

$$
H\left(\nu^{\prime} \circ \bar{\mu}_{6}\right)=\eta_{5} \circ \bar{\mu}_{6} \quad \text { by }(5.3)
$$

and

$$
\begin{aligned}
H\left(\nu^{\prime} \circ \eta_{6} \circ \mu_{7} \circ \sigma_{16}\right) & =\eta_{5}^{2} \circ \mu_{7} \circ \sigma_{16} & \text { by }(5.3) \\
& =4 \zeta_{5} \circ \sigma_{16} & \text { by }(7.14),
\end{aligned}
$$

we have that

$$
\pi_{23}^{3}=\left\{\nu^{\prime} \circ \bar{\mu}_{6}, \nu^{\prime} \circ \eta_{6} \circ \mu_{7} \circ \sigma_{16}\right\} \cong Z_{2} \oplus Z_{2} .
$$

By (5.6), Theorem 12.7 and by the above result, 


$$
\begin{aligned}
\pi_{24}^{4} & =\left\{E \nu^{\prime} \circ \bar{\mu}_{7}, E \nu^{\prime} \circ \eta_{7} \circ \mu_{8} \circ \sigma_{17}, \nu_{4} \circ \sigma^{\prime} \circ \eta_{14} \circ \mu_{15}, \nu_{4}^{2} \circ \kappa_{10}, \nu_{4} \circ \bar{\mu}_{7}, \nu_{4} \circ \eta_{7} \circ \mu_{8} \circ \sigma_{17}\right\} \\
& \simeq Z_{2} \oplus Z_{2} \oplus Z_{2} \oplus Z_{2} \oplus Z_{2} \oplus Z_{2} .
\end{aligned}
$$

Next consider the exact sequence

$$
\begin{array}{ll} 
& \cdots \longrightarrow \pi_{26}^{9} \stackrel{\Delta}{\longrightarrow} \pi_{24}^{4} \stackrel{E}{\longrightarrow} \pi_{25}^{5} \stackrel{H}{\longrightarrow} \pi_{25}^{9} \stackrel{\Delta}{\longrightarrow} \pi_{23}^{4} \longrightarrow \cdots, \\
\text { where } & \pi_{26}^{9}=\left\{\sigma_{9}^{\circ} \circ \eta_{16} \circ \mu_{17}, \nu_{9} \circ \kappa_{12}, \bar{\mu}_{9}, \eta_{9} \circ \mu_{10} \circ \sigma_{19}\right\} \cong Z_{2} \oplus Z_{2} \oplus Z_{2} \oplus Z_{2} \\
\text { and } & \pi_{25}^{9}=\left\{\sigma_{9} \circ \nu_{16}^{3}, \sigma_{9} \circ \mu_{16}, \sigma_{9} \circ \eta_{16} \circ \varepsilon_{17}, \mu_{9} \circ \sigma_{18}\right\} \cong Z_{2} \oplus Z_{2} \oplus Z_{2} \oplus Z_{2} .
\end{array}
$$

We have, by use of Proposition 2.5,

$$
\begin{array}{rlrl}
\Delta\left(\sigma_{9} \circ \eta_{16} \circ \mu_{17}\right) & =\Delta\left(\sigma_{9} \circ \eta_{16}\right) \circ \mu_{15} & \\
& =\left(\nu_{4} \circ \sigma^{\prime} \circ \eta_{14}+E \nu^{\prime} \circ \varepsilon_{7}\right) \circ \mu_{15} & & \text { by }(7.16) \\
& =\nu_{4} \circ \sigma^{\prime} \circ \eta_{14} \circ \mu_{15}+E \nu^{\prime} \circ \varepsilon_{7} \circ \mu_{15}, & \\
\Delta\left(\nu_{9} \circ \kappa_{12}\right) & & \Delta\left(\nu_{9} \circ \kappa_{10}\right. & \\
& =\left( \pm 2 \nu_{4}^{2}\right) \circ \kappa_{10} & & \text { by }(5.13) \\
& =\nu_{4} \circ\left( \pm 2 \nu_{7} \circ \kappa_{10}\right) & & \\
& =0, & & \text { by Theorem } 12.7 \\
& =\Delta\left(\iota_{9}\right) \circ \bar{\mu}_{7} & \\
\Delta\left(\bar{\mu}_{9}\right) & = \pm\left(2 \nu_{4}-E \nu^{\prime}\right) \circ \bar{\mu}_{7} & & \text { by }(5.8) \\
& =\nu_{4} \circ\left( \pm 2 \bar{\mu}_{7}\right)+E \nu^{\prime} \circ\left( \pm \bar{\mu}_{7}\right) & \\
& =E \nu^{\prime} \circ \bar{\mu}_{7}, & \\
\Delta\left(\eta_{9} \circ \mu_{10} \circ \sigma_{19}\right) & =\Delta\left(\eta_{9}\right) \circ \mu_{8} \circ \sigma_{17} & \\
& =E \nu^{\prime} \circ \eta_{7} \circ \mu_{8} \circ \sigma_{17}, & & \text { by }(5.11) .
\end{array}
$$

And in the page 150 we have the following results:

$$
\begin{aligned}
& \Delta\left(\sigma_{9} \circ \nu_{16}^{3}\right)=\nu_{4} \circ \eta_{7} \circ \bar{\varepsilon}_{8}+E\left(\varepsilon^{\prime} \circ \nu_{13}^{3}\right), \\
& \Delta\left(\sigma_{9} \circ \mu_{16}\right)=\nu_{4} \circ \sigma^{\prime} \circ \mu_{14}+E\left(\varepsilon^{\prime} \circ \mu_{13}\right), \\
& \Delta\left(\sigma_{9} \circ \eta_{16} \circ \varepsilon_{17}\right)=\nu_{4} \circ E \zeta^{\prime}+E\left(\varepsilon^{\prime} \circ \eta_{13} \circ \varepsilon_{14}\right), \\
& \Delta\left(\mu_{9} \circ \sigma_{18}\right)=E \nu^{\prime} \circ \mu_{7} \circ \sigma_{16} .
\end{aligned}
$$

Since $\sigma_{9} \circ \nu_{16}^{3}, \sigma_{9} \circ \mu_{16}, \sigma_{9} \circ \eta_{16} \circ \varepsilon_{17}$ and $\mu_{9} \circ \sigma_{18}$ span $\pi_{25}^{9}$ and their images under $\Delta$ are independent, the kernel of $\Delta: \pi_{25}^{9} \rightarrow \pi_{23}^{4}$ is zero. From these facts we have

$$
\pi_{25}^{5}=\left\{\nu_{5}^{2} \circ \kappa_{11}, \nu_{5} \circ \bar{\mu}_{8}, \nu_{5} \circ \eta_{8} \circ \mu_{9} \circ \sigma_{18}\right\} \cong Z_{2} \oplus Z_{2} \oplus Z_{2} .
$$

We have seẹn alsso 


$$
\operatorname{Ker} \Delta\left(: \pi_{26}^{9} \rightarrow \pi_{24}^{4}\right)=\left\{\nu_{9} \circ \kappa_{12}\right\} \approx Z_{2} .
$$

By (5.9), $E\left(\nu_{5} \circ \eta_{8} \circ \mu_{9} \circ \sigma_{18}\right)=0$. Thus $0 \neq \nu_{5} \circ \eta_{8} \circ \mu_{9} \circ \sigma_{18} \in \operatorname{Ker} E=$ $\Delta_{\pi 27}^{11}$. Since $\pi_{27}^{11} \cong Z_{2}$ is generated by $\sigma_{11} \circ \mu_{18}$, we have

$$
\begin{gathered}
\Delta \pi_{27}^{11}=\left\{\Delta\left(\sigma_{11} \circ \mu_{18}\right)\right\}=\left\{\nu_{5} \circ \eta_{8} \circ \mu_{9} \circ \sigma_{18}\right\}, \\
E \pi_{25}^{5}=\left\{\nu_{6}^{2} \circ \kappa_{12}, \nu_{6} \circ \bar{\mu}_{9}\right\} \cong Z_{2} \oplus Z_{2} .
\end{gathered}
$$

We have that the $\Delta$-image of $\pi_{: 6}^{11}=\left\{E^{2} \rho^{\prime}, \bar{\varepsilon}_{11}\right\} \cong Z_{16} \oplus Z_{2}$ is trivial by (12.15). So we obtain an exact sequence

$$
0 \longrightarrow E \pi_{25}^{5} \longrightarrow \pi_{26}^{6} \stackrel{H}{\longrightarrow} \pi_{26}^{11} \longrightarrow 0 \text {. }
$$

Since

$$
\begin{aligned}
\Delta\left(\bar{\varepsilon}_{13}\right) & =\Delta\left(\eta_{13} \circ \kappa_{14}\right) & & \text { by }(15.3) \\
& =\Delta\left(\eta_{13}\right) \circ \kappa_{12} & & \text { by Proposition } 2.5 \\
& =\Delta\left(H\left(\sigma^{\prime}\right)\right) \circ \kappa_{12} & & \text { by Lemma } 5.14 \\
& =0, & &
\end{aligned}
$$

the image of $\Delta: \pi_{28}^{13} \rightarrow \pi_{26}^{6}$ is generated by $\Delta \rho_{13}$.

As

$$
\begin{array}{rlrl}
H\left(\Delta\left(2 \rho_{13}\right)\right) & =H\left(\Delta\left(E^{4} \rho^{\prime}\right)\right) & & \text { by Lemma } 10.9 \\
& = \pm 2 E^{2} \rho^{\prime} & & \text { by Proposition } 2.7, \\
H\left(\Delta \rho_{13}\right) \equiv \pm E^{2} \rho^{\prime} & \bmod \left\{8 E^{2} \rho^{\prime}, \bar{\varepsilon}_{11}\right\} .
\end{array}
$$

So $H\left(\Delta\left(8 \rho_{13}\right)\right)= \pm 8 E^{2} \rho^{\prime} \neq 0$ and $H\left(\Delta\left(16 \rho_{13}\right)\right)= \pm 16 E^{2} \rho^{\prime}=0$.

i.e.,

$$
\Delta\left(16 \rho_{13}\right) \in \text { kernel of } H=E \pi_{25}^{5} \text {. }
$$

Therefore $\operatorname{Im} \Delta \cap \operatorname{Ker} H=\operatorname{Im} \Delta \cap \operatorname{Im} E$ is generated by $\Delta\left(16 \rho_{13}\right)$. Obviously $2 \Delta\left(16 \rho_{13}\right)=\Delta\left(32 \rho_{13}\right)=\Delta(0)=0$. By the definition of $\bar{\mu}_{n}$,

$$
\begin{aligned}
E\left(\nu_{6} \circ \bar{\mu}_{9}\right) & =\nu_{7} \circ \bar{\mu}_{10}=\nu_{7} \circ E^{7}\left\{\mu_{3}, 2 \iota_{12}, 8 \sigma_{12}\right\} & & \\
& =\left\{\nu_{7}, \mu_{10}, 2 \iota_{19}\right\}_{7} \circ 8 \sigma_{20} & & \text { by Proposition } 1.4 \\
& <\pi_{20}^{7} \circ 8 \sigma_{20}=0 & & \text { by Theorem } 7.7 .
\end{aligned}
$$

Thus $\nu_{6} \circ \bar{\mu}_{9} \in \Delta \pi_{27}^{11} \cap E \pi_{25}^{5}$. As $\nu_{6} \circ \bar{\mu}_{9} \neq=0$, we have

$$
\nu_{6} \circ \bar{\mu}_{9}=16 \Delta \rho_{13} \text {. }
$$

That is, the order of $\Delta \rho_{13}$ is 32. In Lemma 15.5 we have chosen such an essential element $\bar{\kappa}^{\prime}$ of $\pi_{26}^{6}$ that $2 \bar{\kappa}^{\prime}=\nu_{6}^{2} \circ \kappa_{12}$. Then we have

and

$$
\pi_{26}^{6}=\left\{\Delta \rho_{13}, \bar{\kappa}^{\prime}\right\} \cong Z_{32} \oplus Z_{4}
$$

$$
E_{\pi_{26}^{6}}^{6}=\left\{E \bar{\kappa}^{\prime}\right\} \cong Z_{4} .
$$


Consider the exact sequence

$$
0 \longrightarrow E \pi_{26}^{6} \longrightarrow \pi_{27}^{7} \stackrel{H}{\longrightarrow} \pi_{27}^{13} \stackrel{\Delta}{\longrightarrow} \pi_{25}^{6} \stackrel{E}{\longrightarrow} \pi_{26}^{7} \longrightarrow \cdots,
$$

where $\pi_{27}^{13}=\left\{\sigma_{13}^{2}, \kappa_{13}\right\} \cong Z_{16} \oplus Z_{2}$. By Theorem 12.9, $\Delta \pi_{27}^{13}=\operatorname{Ker} E=$ $\left\{2 \bar{\sigma}_{6}\right\} \cong Z_{16}$. Thus $H \pi_{27}^{7}=\operatorname{Ker} \Delta \simeq Z_{2}$, and we obtain the exact sequence

$$
0 \longrightarrow E_{\pi_{26}^{6}}^{6} \longrightarrow \pi_{27}^{7} \longrightarrow Z_{2} \longrightarrow 0,
$$

where $E \pi_{26}^{6} \cong Z_{4}$.

Here

$$
\begin{aligned}
4 \bar{\kappa}_{7} & =\nu_{7}^{2} \circ \kappa_{13} & & \text { by Lemma } 15.4 \\
& =2 E \bar{\kappa}^{\prime} & & \text { by Lemma } 15.3 \\
& \neq 0 . & &
\end{aligned}
$$

Therefore the order of $\bar{\kappa}_{7}$ is 8 and

$$
\pi_{27}^{7}=\left\{\bar{\kappa}_{7}\right\} \cong Z_{8} \text {. }
$$

Remark that

$$
2 \bar{\kappa}_{7}= \pm E \bar{\kappa}^{\prime}
$$

We obtain immediately

$$
\pi_{28}^{8}=\left\{\tilde{\kappa}_{8}\right\} \cong Z_{8} \quad \text { by }(5.15) \text { and } \pi_{28}^{15}=0 .
$$

Since $\pi_{29}^{17}=\pi_{30}^{17}=0, E: \pi_{28}^{8} \rightarrow \pi_{29}^{9}$ is an isomorphism onto. Namely,

$$
\pi_{29}^{9}=\left\{\bar{\kappa}_{9}\right\} \cong Z_{8} .
$$

Lemma 16. 1. There exists an element $\beta^{\prime}$ of $\pi_{30}^{10}$ such that

$$
E \beta^{\prime}=\theta^{\prime} \circ \varepsilon_{23}, \quad H\left(\beta^{\prime}\right)=\zeta_{19}, \quad \pm 2 \beta^{\prime}=\Delta\left(\zeta_{21}\right)
$$

and the order of $\beta^{\prime}$ is 8 .

Proof. By (i) of Proposition 11.11 in the case that $i=29$, $n=9$ and $\alpha=\eta_{18} \circ \varepsilon_{19} \in \pi_{27}^{18}$, it follows the existence of $\beta$ such that

$$
E^{2}(\beta)=د\left(\eta_{25} \circ \varepsilon_{26}\right) \text { and } H(\beta) \in\left\{2 \iota_{19}, \eta_{19}, \eta_{20} \circ \varepsilon_{21}\right\}_{2} .
$$

On the other hand

$$
\begin{aligned}
E\left(\theta^{\prime} \circ \varepsilon_{23}\right) & =E \theta^{\prime} \circ \varepsilon_{24} & & \\
& =\Delta \eta_{25} \circ \varepsilon_{24} & & \text { by }(7.30) \\
& =\Delta\left(\eta_{25} \circ \varepsilon_{2 i}\right)=E^{2} \beta & & \text { by Proposition } 2.5 .
\end{aligned}
$$


As

$$
\begin{aligned}
\Delta \pi_{33}^{23} & =\left\{\Delta\left(\eta_{23} \circ \mu_{24}\right)\right\} & & \text { by Theorem } 7.3 \\
& =\left\{\Delta\left(H\left(\theta \circ \mu_{24}\right)\right)\right\} & & \text { by Lemma } 7.5 \\
& =0, & &
\end{aligned}
$$

$E: \pi_{31}^{11} \rightarrow \pi_{32}^{12}$ is a monomorphism.

Thus

$$
\theta^{\prime} \circ \varepsilon_{23}=E \beta \text {. }
$$

Next we consider the secondary composition $\left\{2 \iota_{19}, \eta_{19}, \eta_{20} \circ \varepsilon_{21}\right\}_{2}$.

$H(\beta) \in\left\{2 \iota_{19}, \eta_{19}, \eta_{20} \circ \varepsilon_{21}\right\} \equiv \zeta_{19}, \quad \bmod 2 \pi_{30}\left(S^{19}\right)$ by Lemma 9.1.

So $H(\beta) \equiv \zeta_{19} \quad \bmod 2 \pi_{30}\left(S^{19}\right)+\pi_{21}^{19} \circ \eta_{21} \circ \varepsilon_{22}$

$$
\begin{aligned}
& =2 \pi_{30}\left(S^{19}\right)+\left\{\eta_{19}^{3} \circ \varepsilon_{22}\right\} \\
& =2 \pi_{30}\left(S^{19}\right)+\left\{4 \nu_{19} \circ \varepsilon_{22}\right\} \quad \text { by }(5.5) \\
& =2 \pi_{30}\left(S^{19}\right) \quad \text { by Lemma 6.1. }
\end{aligned}
$$

For some odd integer $r, H(r \beta)=\zeta_{19}$. Set $\beta^{\prime}=r \beta$, then $E \beta^{\prime}=r E \beta$ $=r\left(\theta^{\prime} \circ \varepsilon_{23}\right)=\theta^{\prime} \circ \varepsilon_{23}$, because $2 \varepsilon_{23}=0$. We have $E\left(2 \beta^{\prime}\right)=2 E \beta^{\prime}=2\left(\theta^{\prime} \circ \varepsilon_{23}\right)$ $=\theta^{\prime} \circ\left(2 \varepsilon_{23}\right)=0$. That is, $2 \beta^{\prime} \in \operatorname{Ker} E=\Delta \pi_{32}^{21}=\left\{\Delta \zeta_{21}\right\}$. Therefore $2 \beta^{\prime}$ $=x \Delta \zeta_{21}$ for some integer $x$.

As

$$
\begin{aligned}
8 \beta^{\prime} & =4 x \Delta \zeta_{21} & & \\
& =x \Delta\left(4 \zeta_{21}\right) & & \\
& =x \Delta\left(\eta_{21}^{2} \circ \mu_{23}\right) & & \text { by }(7.14) \\
& =x \Delta\left(H\left(\theta^{\prime} \circ \mu_{23}\right)\right)=0 & & \text { Lemma } 7.5
\end{aligned}
$$

and the order of $H\left(\beta^{\prime}\right)=\zeta_{19}$ is 8 , so the order of $\beta^{\prime}$ is 8 .

Since

$$
\begin{aligned}
2 \zeta_{19} & =H\left(2 \beta^{\prime}\right) \\
& =H\left(x \Delta \zeta_{21}\right) \\
& = \pm 2 x \zeta_{19}
\end{aligned}
$$

by Proposition 2.7 ,

we have

$$
\begin{array}{ll}
2 \equiv \pm 2 x & \bmod 8 \\
1 \equiv \pm x & \bmod 4
\end{array}
$$

Therefore $\Delta \zeta_{21} \equiv \pm 2 \beta^{\prime} \bmod 4\left(2 \beta^{\prime}\right)$, and $8 \beta^{\prime}=0$. We have

$$
\Delta \zeta_{21}= \pm 2 \beta^{\prime}
$$

q.e.d.

Since $\Delta \pi_{30}^{19}=\left\{\Delta \zeta_{19}\right\}=0$ by $(12.22)$ and $\pi_{31}^{19}=0$, we obtain the exact sequence

$$
0 \longrightarrow \pi_{29}^{9} \stackrel{E}{\longrightarrow} \pi_{30}^{10} \stackrel{H}{\longrightarrow} \pi_{30}^{19} \longrightarrow 0,
$$


where $\pi_{29}^{9}=\left\{\bar{\kappa}_{9}\right\} \cong Z_{8}$ and $\dot{\pi}_{30}^{19}=\left\{\zeta_{19}\right\} \cong Z_{8}$ by Theorem 7.4.

By the above lemma, we conclude that this sequence splits. Therefore we have

$$
\pi_{30}^{10}=\left\{\bar{\kappa}_{10}, \beta^{\prime}\right\} \cong Z_{8} \oplus Z_{8} .
$$

Lemma 16. 2. There exists an element $\beta^{\prime \prime}$ of $\pi_{31}^{11}$ such that

$$
\Delta\left(\mu_{25}\right)=E \beta^{\prime \prime}, \quad H\left(\beta^{\prime \prime}\right)=\eta_{21} \circ \mu_{22} \text { and } 2 \beta^{\prime \prime}=0 .
$$

Proof. Let $i=30, n=10$ and $\alpha=\mu_{20}$ in (i) of Proposition 11.10. Then the existence of $\beta^{\prime \prime}$ such that $E \beta^{\prime \prime}=\Delta\left(\mu_{25}\right)$ and $H\left(\beta^{\prime \prime}\right)=\eta_{21} \circ \mu_{22}$ follows immediately. Since the order of $\mu_{25}$ is 2 , we have

$$
2 E \beta^{\prime \prime}=2 \Delta \mu_{25}=\Delta\left(2 \mu_{25}\right)=0 .
$$

$E: \pi_{31}^{11} \rightarrow \pi_{32}^{12}$ is a monomorphism by (16.8). Therefore $2 \beta^{\prime \prime}=0$. q.e.d.

In the exact sequence

$$
\cdots \longrightarrow \pi_{32}^{21} \stackrel{\Delta}{\longrightarrow} \pi_{30}^{10} \stackrel{E}{\longrightarrow} \pi_{31}^{11} \stackrel{H}{\longrightarrow} \pi_{31}^{21} \longrightarrow \cdots,
$$

$\pi_{32}^{21}=\left\{\zeta_{21}\right\} \cong Z_{8}$ and $\pi_{31}^{21}=\left\{\eta_{21} \circ \mu_{22}\right\} \cong Z_{2}$. We have that $\Delta \pi_{32}^{21}=\left\{2 \beta^{\prime}\right\}$ $\cong Z_{4}$ by Lemma $16.1, H$ is an epimorphism, and we have the following result by Lemma 16.2 :

$$
\pi_{31}^{11}=\left\{\bar{\kappa}_{11}, \theta^{\prime} \circ \varepsilon_{23}, \beta^{\prime \prime}\right\} \cong Z_{8} \oplus Z_{2} \oplus Z_{2} .
$$

Lemma 16. 3. There exists an element $\beta^{\prime \prime \prime} \in \pi_{32}^{12}$ such that

$$
E^{2} \beta^{\prime \prime \prime}=8 \Delta \sigma_{29}, \quad H\left(\beta^{\prime \prime \prime}\right)=\mu_{23} \quad \text { and } 2 \beta^{\prime \prime \prime}=0 .
$$

Proof. Consider (ii) of Proposition 11.11 in the case that $n=11, i=31$ and $\alpha=8 \sigma_{22} \in \pi_{29}^{22}$, then we easily obtain an element $\beta$ of $\pi_{32}^{12}$ such that $E^{2} \beta=8 \Delta \sigma_{29}$ and $H(\beta) \in\left\{\eta_{23}, 2 \iota_{24}, 8 \sigma_{24}\right\}_{2}$.

Here $\quad\left\{\eta_{23}, 2 \iota_{24}, 8 \sigma_{24}\right\}_{2} \equiv \mu_{23} \bmod G$ by Lemma 6.5, where $G=\eta_{23} \circ E^{2} \pi_{30}^{22}+\pi_{25}^{23} \circ 8 \sigma_{25}$

$$
\begin{array}{ll}
=\left\{\eta_{23} \circ \varepsilon_{24}, \eta_{23} \bar{\nu}_{24}\right\} & \text { by Theorem } 7.1 \text { aad 7.2 } \\
=\left\{\eta_{23} \circ \varepsilon_{24}, \nu_{23}^{3}\right\} & \text { by Lemma 6.3. }
\end{array}
$$

That is, $H(\beta) \equiv \mu_{23} \bmod \left\{\eta_{23} \circ \varepsilon_{24}, \nu_{23}^{3}\right\}$.

As $H\left(\theta \circ \varepsilon_{24}\right)=\eta_{23} \circ \varepsilon_{24}$ by Lemma 7.5 and $H\left(\theta \circ \bar{\nu}_{24}\right)=\eta_{23} \circ \bar{\nu}_{24}=\nu_{23}^{3}$ by Lemma 7.5 and Lemma 6.3 , there exists $\beta^{\prime \prime \prime} \in \pi_{32}^{12}$ such that $\beta^{\prime \prime \prime} \equiv \beta$ $\bmod \left\{\theta \circ \varepsilon_{24}, \theta \circ \bar{\nu}_{24}\right\}$ and $H\left(\beta^{\prime \prime \prime}\right)=\mu_{23}$. Also 


$$
\begin{aligned}
& E^{2} \beta^{\prime \prime \prime} \equiv E^{2} \beta \quad \bmod \left\{E^{2}\left(\theta \circ \varepsilon_{24}\right), \quad E^{2}\left(\theta \circ \bar{\nu}_{24}\right)\right\}=0 \quad \text { by }(7.30), \\
& E^{2} \beta^{\prime \prime \prime}=8 \Delta \sigma_{29} .
\end{aligned}
$$

Next consider the secondary composition $\left\{\sigma_{12}, \nu_{19}, \mu_{22}\right\}_{1}$ which can be defined by (7.20) and (7.25), and choose an element $\gamma$ from this secondary composition. By Proposition 2.6,

$$
\begin{array}{ccc}
H(\gamma) \in H\left\{\sigma_{12}, \nu_{19}, \mu_{22}\right\}_{1} & =-\Delta^{-1}\left(\sigma_{11} \circ \nu_{18}\right) \circ \mu_{23} \\
& \ni-\iota_{23} \circ \mu_{23}=\mu_{23} & \text { by }(7.21), \\
\text { i.e., } & \bmod H\left(\sigma_{12} \circ \pi_{32}^{19}+\pi_{23}^{12} \circ \mu_{23}\right)=0 . \mu_{23} & \bmod
\end{array}
$$

As $H\left(\gamma-\beta^{\prime \prime \prime}\right)=\mu_{23}-\mu_{23}=0, \quad \gamma-\beta^{\prime \prime \prime} \in E \pi_{31}^{11}=\left\{\bar{\kappa}_{12}, E \theta^{\prime} \circ \varepsilon_{24}, E \beta^{\prime \prime}\right\}$.

Here $2 \gamma \in\left\{\sigma_{12}, \nu_{19}, \mu_{22}\right\}_{1} \circ 2 \iota_{32}=\sigma_{12} \circ E\left\{\nu_{18}, \mu_{21}, 2 \iota_{30}\right\}$

$$
\text { C } \sigma_{12} \circ \pi_{32}^{19}=0 \quad \text { by Theorem } 6.7 \text {, }
$$

$$
\begin{aligned}
2\left(\gamma-\beta^{\prime \prime \prime}\right)=-2 \beta^{\prime \prime \prime} \in 2 E \pi_{31}^{11} & =\left\{2 \bar{\kappa}_{12}, 2\left(E \theta^{\prime} \circ \varepsilon_{24}\right), 2 E \beta^{\prime \prime}\right\} \\
& =\left\{2 \bar{\kappa}_{12}, E \theta^{\prime} \circ\left(2 \varepsilon_{24}\right), \Delta\left(2 \mu_{25}\right)\right\} \\
& =\left\{2 \bar{\kappa}_{12}\right\} \quad \text { by Lemmas 6.1, 6.5. }
\end{aligned}
$$

Therefore $2 \beta^{\prime \prime \prime}=2 x \bar{\kappa}_{12}$ for some integer $x$.

The kernel of $E^{2}: \pi_{32}^{12} \rightarrow \pi_{34}^{14}$ is generated by $E \theta^{\prime} \circ \varepsilon_{24}, \Delta \mu_{25}, \theta \circ \bar{\nu}_{24}$ and $\theta \circ \varepsilon_{24}$, since $\pi_{34}^{25}=\left\{\eta_{25} \circ \varepsilon_{26}, \nu_{25}^{3}, \mu_{25}\right\}, \pi_{35}^{27}=\left\{\bar{\nu}_{27}, \varepsilon_{27}\right\}$ and the following relations hold :

$$
\begin{array}{lll}
(16.10) & \Delta\left(\bar{\nu}_{27}\right)=\Delta\left(\iota_{27}\right) \circ \bar{\nu}_{25}=E \theta \circ \bar{\nu}_{25} & \text { by }(7.30), \\
& \Delta\left(\varepsilon_{27}\right)=\Delta\left(\iota_{27}\right) \circ \varepsilon_{25}=E \theta \circ \varepsilon_{25} & \text { by }(7.30) \\
(16.11) & \Delta\left(\eta_{25} \circ \varepsilon_{26}\right)=\Delta\left(\eta_{25}\right) \circ \varepsilon_{24}=E \theta^{\prime} \circ \varepsilon_{24} & \text { by }(7.30) \\
& \Delta\left(\nu_{25}^{3}\right)=\Delta\left(\nu_{25}^{2}\right) \circ \nu_{29}=\Delta(H(\lambda)) \circ \nu_{29}=0 & \text { by Lemma 12.18. }
\end{array}
$$

As $\quad 2 x \bar{\kappa}_{14}=E^{2}\left(2 x \bar{\kappa}_{12}\right)=E^{2}\left(2 \beta^{\prime \prime \prime}\right)=16 \Delta \sigma_{29}=\Delta\left(16 \sigma_{29}\right)=0$, so $2 x \bar{\kappa}_{12}=y\left(E \theta^{\prime} \circ \varepsilon_{24}\right)+z\left(\Delta \mu_{25}\right)+u\left(\theta \circ \bar{\nu}_{24}\right)+v\left(\theta \circ \varepsilon_{24}\right)$

by the above facts, where $y, z, u, v=0,1$. Operating $H$ we know $u=v=0$. Then

By (16.8)

$$
\begin{aligned}
2 x \bar{\kappa}_{12} & =y\left(E \theta^{\prime} \circ \varepsilon_{24}\right)+z\left(\Delta \mu_{25}\right) \\
& =y E\left(\theta^{\prime} \circ \varepsilon_{23}\right)+z E \beta^{\prime \prime} .
\end{aligned}
$$

Since $\bar{\kappa}_{11}, \theta^{\prime} \circ \varepsilon_{23}$ and $\beta^{\prime \prime}$ are independent generators of $\pi_{31}^{11}$, we have $y=z=0$. Thus $2 \beta^{\prime \prime \prime}=2 x \bar{\kappa}_{12}=0$.

q.e.d. 
In the exact sequence $\cdots \rightarrow \pi_{33}^{23} \stackrel{\Delta}{\longrightarrow} \pi_{31}^{11} \stackrel{E}{\longrightarrow} \pi_{32}^{12} \stackrel{H}{\longrightarrow} \pi_{32}^{23} \rightarrow \cdots$, $E$ is a monomorphism by (16.8) and $H$ is an epimorphism, since $\pi_{32}^{23}$ is generated by $\nu_{23}^{3}, \mu_{23}$ and $\eta_{23} \circ \varepsilon_{24}$, and

$$
\begin{array}{ll}
H\left(\theta \circ \bar{\nu}_{24}\right)=\eta_{23} \circ \bar{\nu}_{24}=\nu_{23}^{3} & \text { by Lemma } 7.5, \\
H\left(\theta \circ \varepsilon_{24}\right)=\eta_{23} \circ \varepsilon_{24} & \text { by Lemma } 7.5, \\
H\left(\beta^{\prime \prime \prime}\right)=\mu_{23} & \text { by Lemma } 16.3 .
\end{array}
$$

Obviously the order of $\theta \circ \bar{\nu}_{24}, \theta \circ \varepsilon_{24}$ and $\beta^{\prime \prime \prime}$ is 2 . So the following exact sequence splits :

$$
0 \longrightarrow \pi_{31}^{11} \stackrel{E}{\longrightarrow} \pi_{32}^{12} \stackrel{H}{\longrightarrow} \pi_{32}^{23} \longrightarrow 0 \text {. }
$$

Therefore $\quad \pi_{32}^{12}=\left\{\bar{\kappa}_{12}, E \theta^{\prime} \circ \varepsilon_{24}, \Delta \mu_{25}, \theta \circ \varepsilon_{24}, \theta \circ \bar{\nu}_{24}, \beta^{\prime \prime \prime}\right\}$

$$
\cong Z_{8} \oplus Z_{2} \oplus Z_{2} \oplus Z_{2} \oplus Z_{2} \oplus Z_{2} \text {. }
$$

Consider the exact sequence

$$
\cdots \longrightarrow \pi_{34}^{25} \stackrel{\Delta}{\longrightarrow} \pi_{32}^{12} \stackrel{E}{\longrightarrow} \pi_{33}^{13} \stackrel{H}{\longrightarrow} \pi_{33}^{25} \stackrel{\Delta}{\longrightarrow} \pi_{31}^{12} \longrightarrow \cdots,
$$

where $\pi_{34}^{25}=\left\{\nu_{25}^{3}, \mu_{25}, \eta_{25} \circ \varepsilon_{26}\right\}$ and $\pi_{33}^{25}=\left\{\bar{\nu}_{25}, \varepsilon_{25}\right\}$.

By (12.26), $\Delta: \pi_{33}^{25} \rightarrow \pi_{31}^{12}$ is a monomorphism, so $H$ is trivial. Therefore we obtain

$$
\pi_{33}^{13}=E_{\pi_{32}^{12}}^{12}=\left\{\bar{\kappa}_{13}, E \theta \circ \varepsilon_{25}, E \theta \circ \bar{\nu}_{25}, E \beta^{\prime \prime \prime}\right\} \simeq Z_{8} \oplus Z_{2} \oplus Z_{2} \oplus Z_{2},
$$

by (16.11).

As $\Delta \pi_{34}^{27}=\left\{\Delta \sigma_{27}\right\}=\left\{\xi_{13} \circ \eta_{31}\right\}$ by (12.26), we have $H \pi_{34}^{14}=\left\{2 \sigma_{27}\right\} \cong Z_{8}$ in the exact sequence

$$
\cdots \longrightarrow \pi_{35}^{27} \stackrel{\Delta}{\longrightarrow} \pi_{33}^{13} \stackrel{E}{\longrightarrow} \pi_{34}^{14} \stackrel{H}{\longrightarrow} \pi_{34}^{27} \stackrel{\Delta}{\longrightarrow} \pi_{32}^{13} \longrightarrow \cdots,
$$

where $\pi_{35}^{27}=\left\{\bar{\nu}_{27}, \varepsilon_{27}\right\}$ and $\pi_{34}^{27}=\left\{\sigma_{27}\right\} \cong Z_{16}$.

By the use of (16.10), $E_{\pi_{33}^{13}}^{13}=\left\{\bar{\kappa}_{14}, E^{2}{\beta^{\prime \prime \prime}}^{\prime \prime} \simeq Z_{8} \oplus Z_{2}\right.$.

Then, we have obtained the exact sequence

$$
0 \longrightarrow\left\{\bar{\kappa}_{13}, E \beta^{\prime \prime \prime}\right\} \stackrel{E}{\longrightarrow} \pi_{34}^{14} \stackrel{H}{\longrightarrow}\left\{2 \sigma_{27}\right\} \longrightarrow 0 .
$$

Since $8 \Delta \sigma_{29}=E^{2} \beta^{\prime \prime \prime} \neq 0$ by Lernma 16.3 , the order of $\Delta \sigma_{29}$ is 16 . We have also $H \Delta \sigma_{29}= \pm 2 \sigma_{27}$. Therefore 
The $(n+20)-t h$ homotopy groups of $n$-spheres

$$
\pi_{34}^{14}=\left\{\Delta \sigma_{29}, \bar{\kappa}_{14}\right\}=Z_{16} \oplus Z_{8} .
$$

Consider the exact sequence

$$
\cdots \longrightarrow \pi_{36}^{29} \stackrel{\Delta}{\longrightarrow} \pi_{34}^{14} \stackrel{E}{\longrightarrow} \pi_{35}^{15} \stackrel{H}{\longrightarrow} \pi_{35}^{29} \stackrel{\Delta}{\longrightarrow} \pi_{33}^{14} \longrightarrow \cdots,
$$

where $\pi_{36}^{29}=\left\{\sigma_{29}\right\}, \pi_{35}^{29}=\left\{\nu_{29}^{2}\right\}$ and $\pi_{33}^{14}=\left\{\omega_{14} \circ \nu_{30}, \bar{\sigma}_{14}, \bar{\zeta}_{14}\right\}$.

Since $\Delta\left(\nu_{29}^{2}\right)=E^{2} \omega^{\prime}=2\left(\omega_{14} \circ \nu_{30}\right) \neq 0$ by $(12.27)$, the above $H$ is trivial. It is obvious that the kernel of $E: \pi_{34}^{14} \rightarrow \pi_{35}^{15}$ is generated by $\Delta \sigma_{29}$. Thus we obtain $\pi_{35}^{15}=\left\{\bar{\kappa}_{15}\right\} \cong Z_{8}$.

$\Delta\left(\nu_{31}^{2}\right)=\Delta\left(H\left(\nu_{16}^{*} \circ \nu_{34}\right)\right)=0$ by Lemma 12.16 , where $\nu_{31}^{2}$ is a generator of $\pi_{37}^{31}$. We have also $\pi_{36}^{31}=0$. Thus $E: \pi_{35}^{15} \rightarrow \pi_{36}^{16}$ is an isomorphism onto. Then

$$
\pi_{36}^{16}=\left\{\bar{\kappa}_{16}\right\} \cong Z_{8} .
$$

Next $\pi_{37}^{17}=\left\{\bar{\kappa}_{17}\right\} \cong Z_{8}$ follows immediately from $\pi_{38}^{33}=\pi_{37}^{33}=0$.

Lemma 16.4. There exists an element $\bar{\beta}$ of $\pi_{39}^{19}$ such that

$$
E \bar{\beta}=\Delta \eta_{41} \quad \text { and } \quad H(\bar{\beta})=\eta_{37}^{2} .
$$

Proof. This follows immediately if we take $n=18, i=38$ and $\alpha=\eta_{36} \in \pi_{37}^{36}$ in (ii) of Proposition 11.10.

Now consider the exact sequence

$$
\cdots \longrightarrow \pi_{39}^{35} \stackrel{\Delta}{\longrightarrow} \pi_{37}^{17} \stackrel{E}{\longrightarrow} \pi_{38}^{18} \stackrel{H}{\longrightarrow} \pi_{38}^{35} \stackrel{\Delta}{\longrightarrow} \pi_{36}^{17} \longrightarrow \cdots,
$$

where $\pi_{39}^{35}=0, \pi_{38}^{25}=\left\{\nu_{35}\right\}$ and $\pi_{36}^{17}=\left\{\omega_{17} \circ \nu_{33}, \bar{\sigma}_{17}, \bar{\zeta}_{17}\right\}$.

$H \pi_{38}^{13}=\left\{2 \nu_{35}\right\} \cong Z_{4}$, since $\Delta \nu_{35}=\omega_{17} \circ \nu_{33}$ and $\Delta\left(2 \nu_{35}\right)=2\left(\omega_{17} \circ \nu_{33}\right)=0$ (see page 170).

$H\left(\Delta \nu_{37}\right)= \pm 2 \nu_{15}$ by Proposition 2.7. Thus $2 \Delta \nu_{37}=1-0$ in $\pi_{38}^{18}$.

By the above Lemma 16.4 and (5.5),

$$
4 \Delta \nu_{37}=\Delta\left(\eta_{37}^{3}\right)=\Delta\left(H\left(\bar{\beta}_{\circ} \eta_{39}\right)\right)=0 .
$$

i.e., the order of $\Delta \nu_{37}$ is 4 . Therefore the following exact sequence splits :

and

$$
0 \longrightarrow \pi_{37}^{17} \stackrel{E}{\longrightarrow} \pi_{38}^{18} \stackrel{H}{\longrightarrow}\left\{2 \nu_{35}\right\} \longrightarrow 0
$$

$$
\pi_{38}^{18}=\left\{\bar{\kappa}_{18}, \Delta \nu_{37}\right\} \cong Z_{8} \oplus Z_{4} \text {. }
$$


Lemma 16. 5. There exists an element $\overline{\bar{\beta}}$ of $\pi_{40}^{20}$ such that

$$
E \overline{\bar{\beta}}=\Delta \iota_{43} \quad \text { and } \quad H(\overline{\bar{\beta}})=\eta_{39} \text {. }
$$

Proof. This lemma immediately follows if we take $n=19$, $i=39$ and $\alpha=\iota_{38}$ in (ii) of Proposition 11.10.

Now we note that $E: \pi_{39}^{19} \rightarrow \pi_{40}^{20}$ is a monomorphism, for, in the exact sequence $\pi_{41}^{39} \stackrel{\Delta}{\longrightarrow} \pi_{39}^{19} \stackrel{E}{\longrightarrow} \pi_{40}^{20}$, the generator $\eta_{39}^{2}$ of $\pi_{41}^{39}$ is an $H$-image of $\bar{\beta} \circ \eta_{40}$. Since $2 E \bar{\beta}=2 \Delta \eta_{41}=\Delta\left(2 \eta_{41}\right)=0$ and $E: \pi_{39}^{19} \rightarrow \pi_{40}^{20}$ is a monomorphism, the order of $\bar{\beta}$ in Lemma 16.4 is 2 . Therefore the following exact sequence splits :

$$
0 \longrightarrow E_{38}^{18} \longrightarrow \pi_{39}^{19} \stackrel{H}{\longrightarrow} \pi_{39}^{37} \longrightarrow 0,
$$

where $E_{\pi_{38}^{18}}^{18}=\left\{\bar{\kappa}_{19}\right\} \cong Z_{8}$ and $\pi_{39}^{37}=\left\{\eta_{37}^{2}\right\}$.

By the use of Lemma 16.4,

$$
\pi_{39}^{19}=\left\{\bar{\kappa}_{19}, \bar{\beta}\right\} \cong Z_{8} \oplus Z_{2} .
$$

Since $\Delta\left(\eta_{39}\right)=0$ and $\Delta\left(\eta_{39}^{2}\right)=0$ by Lemma 16.5 , we have an exact sequence

$$
0 \longrightarrow \pi_{39}^{19} \stackrel{E}{\longrightarrow} \pi_{40}^{20} \stackrel{H}{\longrightarrow} \pi_{40}^{39} \longrightarrow 0 .
$$

Lemma 16. 6. The above exact sequence splits, that is, the order of $\overline{\bar{\beta}}$ in Lemma 16.5 is 2.

Proof. It follows from the homotopy exact sequence associated with the pair $\left(\Omega^{2}\left(S^{20}\right), S^{18}\right)$ that the following sequence is exact:

$$
\cdots \rightarrow \pi_{38}^{18} \rightarrow \pi_{40}^{20} \rightarrow \pi_{38}\left(\left(\Omega^{2}\left(S^{20}\right), S^{18}\right): 2\right) \rightarrow \pi_{37}^{18} \rightarrow \cdots,
$$

where $\pi_{38}^{18}=\left\{\bar{\kappa}_{18}, \Delta \nu_{37}\right\} \cong Z_{8} \oplus Z_{4}, \pi_{37}^{18}=\left\{\bar{\zeta}_{18}, \bar{\sigma}_{18}\right\} \cong Z_{8} \oplus Z_{2}$ and $\pi_{38}\left(\left(\Omega^{2}\left(S^{20}\right)\right.\right.$, $\left.S^{18}\right): 2$ ) denotes the 2-primary component of $\pi_{38}\left(\Omega^{2}\left(S^{20}\right), S^{18}\right)$.

By Theorem 11.7 in the case that $i=37, n=18$ and $k=2$,

$$
\pi_{38}\left(\left(\Omega^{2}\left(S^{20}\right), S^{18}\right): 2\right) \cong \pi_{37}\left(E^{17} P_{18}^{19}: 2\right) .
$$

Here $P_{18}^{19}=S^{18} \vee e^{19}$ which is homotopy equivalent to $S^{18} \vee S^{19}$. For, the attaching map of $e^{19}$ is trivial since 18 is even.

Therefore 


$$
\begin{aligned}
\pi_{18}\left(\left(\Omega^{2}\left(S^{20}\right), S^{18}\right): 2\right) & \simeq \pi_{37}\left(S^{35} \vee S^{36}: 2\right) \\
& \simeq \pi_{37}^{35} \oplus \pi_{37}^{36} \quad \text { by }(4.18) \text { of }[4] \\
& \simeq Z_{2} \oplus Z_{2} .
\end{aligned}
$$

The element $2 \bar{\beta} \in \pi_{40}^{20}$ is a multiple of $E(\bar{\beta})=\Delta \eta_{41}$, since $E(2 \overline{\bar{\beta}})$ $=2\left(\Delta \iota_{43}\right)=0$ and since $\Delta \eta_{41}$ generates the kernel of $E: \pi_{40}^{20} \rightarrow \pi_{41}^{21}$. So, there are two possibilities : $\pi_{40}^{20} \cong Z_{8} \oplus Z_{4}$ or $\pi_{40}^{20} \cong Z_{8} \oplus Z_{2} \oplus Z_{2}$.

Since $\pi_{38}^{18}=\left\{\bar{\kappa}_{18}, \Delta \nu_{37}\right\} \cong Z_{8} \oplus Z_{4}$ and $E: \pi_{39}^{19} \rightarrow \pi_{40}^{20}$ is a monomorphism, $E^{2} \pi_{38}^{18}=\left\{\bar{\kappa}_{20}\right\} \cong Z_{8}$. Therefore by considering the exact sequence (16.13) the lemma follows.

By Lemma 16.6,

$$
\pi_{40}^{20}=\left\{\bar{\kappa}_{20}, \Delta \eta_{41}, \bar{\beta}\right\} \cong Z_{8} \oplus Z_{2} \oplus Z_{2} .
$$

Since $\Delta: \pi_{41}^{41} \rightarrow \pi_{39}^{20}$ is a monomorphism, we have an exact sequence

$$
0 \longrightarrow E_{\pi_{40}^{20}} \longrightarrow \pi_{41}^{21} \longrightarrow 0,
$$

where $E \pi_{40}^{20}=\left\{\bar{\kappa}_{21}, E \beta\right\} \cong Z_{8} \oplus Z_{2}$. Therefore

$$
\pi_{41}^{21}=\left\{\bar{\kappa}_{21}, \Delta \iota_{43}\right\} \cong Z_{8} \oplus Z_{2} \text {, where } \Delta \iota_{43}=E \overline{\bar{\beta}} \quad \text { by Lemma 16.5. }
$$

As it is obvious that the kernel of $E: \pi_{41}^{21} \rightarrow \pi_{42}^{22}$ is generated by $\Delta \iota_{43}$ and $\pi_{42}^{43}=0$, we obtain $\pi_{42}^{22}=\left\{\bar{\kappa}_{22}\right\} \cong Z_{8}$.

The result for the stable group $\left(G_{20} ; 2\right)=\{\bar{\kappa}\} \cong Z_{8}$ follows immediately.

(ii) Odd primary components.

We shall compute $p$-primary components $\pi_{n+20}\left(S^{n}: p\right)$ for odd prime $p$ in order to complete our calculation of $\pi_{n+20}\left(S^{n}\right)$.

By Serre's isomorphism [1]

$$
\pi_{i-1}\left(S^{2 m-1}: p\right) \oplus \pi_{i}\left(S^{4 m-1}: p\right) \cong \pi_{i}\left(S^{2 m}: p\right)
$$

which is given by the correspondence $(\alpha, \beta) \rightarrow E \alpha+\left[\iota_{2 m}, \iota_{2 m}\right] \circ \beta$, we compute only the groups $\pi_{n+20}\left(S^{n}: p\right)$ for the case that $n$ is odd.

First, there are no odd prime $p$ satisfying

or

$$
\begin{array}{ll}
2 i(p-1)-2=20 & \text { for } i=2, \cdots, p-1, \\
2 i(p-1)-1=20 & \text { for } i=1,2, \cdots, p-1 .
\end{array}
$$

Whence we have the next result by Theorem 13.4. 
(16. 15) $\pi_{2 m+1+20}\left(S^{2 m+1}: p\right)=0$ for $m \geq 1$ and an odd prime $p \geq 5$.

Next we shall compute the 3-primary component of $\pi_{2 m+1+20}\left(S^{2 m+1}\right)$ for $m \geq 1$. We shall use the following results of [2]: (16. 16) 3-primary component of $G_{20}=\left\{\beta_{1} \circ \beta_{2}\right\} \cong Z_{3}$.

For the simplicity we shall use the following notations.

$$
\pi_{n+k}^{n}=\pi_{n+k}\left(S^{n}: 3\right) \quad \text { and } \quad Q_{i}^{n}=\pi_{i}\left(\left(\Omega^{2}\left(S^{n}\right), S^{n-2}\right): 3\right) .
$$

The next two exact sequences are mainly used in this last section.

$$
\cdots \longrightarrow \pi_{i}^{n} \stackrel{E^{2}}{\longrightarrow} \pi_{i+2}^{n+2} \longrightarrow Q_{i}^{n+2} \stackrel{\partial}{\longrightarrow} \pi_{i-1}^{n} \longrightarrow \cdots \text { for } n: \text { odd }
$$

and

$$
\begin{array}{r}
\cdots \longrightarrow \pi_{i+2}^{3(n+1)+1} \stackrel{\Delta}{\longrightarrow} \pi_{i}^{(3 n+1)-1} \longrightarrow Q_{i-1}^{n+2} \longrightarrow \pi_{i+1}^{3(n+1)+1} \longrightarrow \cdots \\
\text { for } i>3(n+1)-1 \text { and } n: \text { odd },
\end{array}
$$

where $\Delta \circ E^{2}=f_{3 *}$ for a mapping $f_{3}: S^{3(n+1)-1} \rightarrow S^{3(n+1)-1}$ of degree 3 .

For the case $n=1$, we have the following exact sequence (see Proposition 13.3)

$$
\cdots \longrightarrow \pi_{i+2}^{7} \stackrel{\Delta}{\longrightarrow} \pi_{i}^{5} \longrightarrow \pi_{i+1}^{3} \longrightarrow \pi_{i+1}^{7} \stackrel{\Delta}{\longrightarrow} \cdots \text { for } i>5,
$$

such that $\Delta \circ E^{2}=f_{3 *}$.

$\pi_{22}^{5}=\pi_{23}^{7}=0$ by Theorem 13. 10, hence we have $\pi_{23}^{3}=0$.

By (13.6)

$$
\begin{array}{ll}
Q_{2 m+1+18}^{2 m+1}=0 & \text { for } m \geq 3, \\
Q_{2 m+1+19}^{2 m+1}=0 & \text { for } m \geq 4 .
\end{array}
$$

By inserting these values in the exact sequence (16.17), we obtain the isomorphism onto for $m \geq 3$ :

$$
E^{2}: \pi_{2 m+1+20}^{2 m+1} \cong \pi_{2 m+3+20}^{2 m+3} \text {. }
$$

So by (16. 16),

$$
\pi_{2 m+1+20}^{2 m+1} \cong Z_{3} \quad \text { for } m \geq 3 .
$$

Consider the exact sequence of $(16.17)$ in the case that $n=3$ and $i=23$, that is

$$
\cdots \longrightarrow \pi_{23}^{3} \stackrel{E^{2}}{\longrightarrow} \pi_{25}^{5} \longrightarrow Q_{23}^{5} \stackrel{\partial}{\longrightarrow} \pi_{22}^{3} \stackrel{E^{2}}{\longrightarrow} \pi_{24}^{5} \longrightarrow \cdots,
$$


where $\pi_{23}^{3}=0$ and $E^{2}: \pi_{22}^{3} \rightarrow \pi_{24}^{5}$ is an isomorphism onto by (vii) of Theorem 13.10. Therefore we have

$$
\pi_{25}^{5} \cong Q_{23}^{5} \text {. }
$$

Next consider the exact sequence (16.18) in the case that $n=3$, $i=21$, that is

$$
\cdots \longrightarrow \pi_{26}^{13} \stackrel{\Delta}{\longrightarrow} \pi_{24}^{11} \longrightarrow Q_{23}^{5} \longrightarrow \pi_{25}^{13} \longrightarrow \cdots,
$$

where $\pi_{26}^{13}=\left\{\alpha_{1}(13) \circ \beta_{1}(16)\right\} \cong Z_{3}, \pi_{24}^{11}=\left\{\alpha_{1}(11) \circ \beta_{1}(14)\right\} \cong Z_{3}$ and $\pi_{25}^{13}=0$ by (i) of Theorem 13.10 and Theorem 13.9. As $\Delta \circ E^{2}=f_{3 *}$,

$$
\Delta\left(\alpha_{1}(13) \circ \beta_{1}(16)\right)=3\left(\alpha_{1}(11) \circ \beta_{1}(14)\right)=0 .
$$

Thus $Q_{23}^{5} \cong \pi_{24}^{11} \cong Z_{3}$. Therefore by $(16.20)$

$$
\pi_{25}^{5} \simeq Z_{3} \text {. }
$$

Summarizing these results, we have the following

Theorem 16. 7.

$$
\begin{aligned}
& \pi_{2 m+1+20}\left(S^{2 m+1}: p\right)=0 \quad \text { for } m \geq 1 \text { and odd prime } p \geq 5 \text {, and } \\
& \pi_{2 m+1+20}\left(S^{2 m+1}: 3\right) \cong \begin{cases}0 & m=1 \\
Z_{3} & m \geq 2\end{cases}
\end{aligned}
$$

For even dimensional spheres, their homotopy groups are easily calculated by use of Serre's isomorphism (16.14) and the known results for $\pi_{2 m+1+k}\left(S^{2 m+1}: p\right)$ where $k \leq 20$, and the results are the following.

Theorem 16. 8. The odd primary components of $\pi_{2 n+20}\left(S^{2 n}\right)$ are isomorphic to

$$
\begin{array}{ll}
Z_{3} & \text { for } n=6,8 \text { and } n \geq 10, \\
Z_{3} \oplus Z_{3} & \text { for } n=4,9, \\
Z_{15} \oplus Z_{3} & \text { for } n=3,7 \\
Z_{33} & \text { for } n=1 \\
Z_{63} \oplus Z_{3} & \text { for } n=5, \\
0 & \text { for } n=2 .
\end{array}
$$




\section{BIBLIOGRAPHY}

[1] J-P. Serre: Groupes d'homotopie et classes des groupes abéliens, Ann. of Math., 58 (1953), 258-294.

[2] H. Toda : p-primary components of homotopy groups IV, Compositions and toric constructions, Memoirs Univ. of Kyoto, 32 (1959), 297-332.

[3] H. Toda : Composition methods in homotopy groups of spheres, Ann. of Math. Studies. No. 49. Princeton (1962).

[4] G. W. Whitehead : A generalization of the Hopf invariant, Ann. of Math., 51 (1950), 192-237. 